\title{
Ultrasound-assisted thrombolysis for a giant right atrial thrombus and pulmonary embolism in a COVID-19 patient
}

\author{
José Roberto Victoria-Nandayapa', Cuitláhuac Arroyo-Rodríguez', Salvador Leopoldo Franco-Rodríguez², \\ Fausto Miguel Pérez-Méndez ${ }^{3}$, Alan Humberto Soto-Gaxiola ${ }^{4}$
}

'Cardiology Department, Hospital San José Hermosillo, Sonora, México

2Radiology Department, Hospital San José Hermosillo, Sonora, México

${ }^{3}$ Pulmonology Department, Hospital San José Hermosillo, Sonora, México

${ }^{4}$ Infectious Diseases Department, Hospital San José Hermosillo, Sonora, México

\section{Correspondence to: Cuitláhuac Arroyo- -Rodríguez, MD, Morelos No. 340, Colonia Bachoco, CP 83148 Hermosillo, Sonora México, phone: +52 6441678937, e-mail: cuitla88@hotmail.com Copyright by the Author(s), 2021 \\ Kardiol Pol. 2021; 79 (6): 710-711: DOI: $10.33963 /$ KP.15961 Received: November 12, 2020 Revision accepted: April 11, 2021 Published online: April 16, 2021}

A 51-year-old male with type-2 diabetes and hypertension was admitted because of SARS-CoV-2 pneumonia (confirmed by real-time polymerase chain reaction). Physical examination revealed tachypnea, room air oxygen saturation $70 \%$, body temperature $37.8^{\circ} \mathrm{C}$, blood pressure $137 / 89 \mathrm{~mm} \mathrm{Hg}$, and a pulse of $100 \mathrm{bpm}$. Chest computed tomography (CT) showed extensive interstitial pneumonia affecting upper lobes with ground-glass opacities and crazy-paving pattern in lower lobes (Figure 1A). Global CTscore was 19 (severe involvement) [1].

Initial D-dimer was $333 \mathrm{ng} / \mathrm{ml}$ (reference range $<500 \mathrm{ng} / \mathrm{ml}$ ), troponin I was $0.003 \mathrm{ng} / \mathrm{ml}$ (reference range $<0.03 \mathrm{ng} / \mathrm{ml}$ ). Thromboprophylaxis with enoxaparin $60 \mathrm{mg}$ daily was initiated.

Two days after admission he progressed to severe acute respiratory distress syndrome and hemodynamic instability requiring invasive mechanical ventilation and vasopressors. D-dimer peaked at $30994 \mathrm{ng} / \mathrm{ml}$, and troponin I reached $0.046 \mathrm{ng} / \mathrm{ml}$. Transthoracic echocardiogram (TTE) showed a large, multilobular, highly mobile mass in the right atrium protruding into the right ventricle (Figure 1B; Supplementary material, Video S1). Right ventricular systolic function was impaired (shortening fraction $27 \%$, tricuspid annular plane systolic excursion $15 \mathrm{~mm}$ ).

Pulmonary $\mathrm{CT}$ angiography revealed a $51 \times 25 \mathrm{~mm}$ filling defect in the right atrium (thrombus) (Figure 1C) and pulmonary embolus in the posterior basal segmental artery (right lung) (Figure 1D). The right ventricular to left ventricular diameter ratio was 1.30 .

Hemodynamic instability, a recent self-limited inadvertent subclavian arterial puncture (relative contraindication for systemic thrombolysis) and concerns about possible incomplete lysis with systemic thrombolysis due to the large size thrombus led to the decision of performing ultrasound-assisted thrombolysis. Before the procedure pulmonary arterial systolic/diastolic/mean pressure was 44/17/26 mm Hg. Invasive pulmonary angiography showed a hypoperfused area in the right lower pulmonary lobe (Figure 1E). The longest EKOS catheter (135 cm length) was chosen, targeting the treatment zone in the right atrium and right pulmonary artery (Figure 1F; Supplementary material, Video S2). Eighteen $\mathrm{mg}$ of alteplase $(0.75 \mathrm{mg} / \mathrm{h})$ were infused over 24 hours. Unfractionated heparin with a target activated partial thromboplastin time of 46 to 70 seconds was given simultaneously to alteplase. Enoxaparin $60 \mathrm{mg}$ twice daily was administaered afterward. The patient improved soon thereafter and was extubated 2 days later. A 24-hour post-procedure TTE showed a $9 \times 2 \mathrm{~mm}$ remnant thrombus in the right atrium. Repeated TTE 5 days post-procedure showed thrombus resolution (Supplementary material, Video S3). The patient was discharged on day 10 post-procedure with apixaban. At 1 month follow-up, he fully reintegrated. to previous life and working activities. However, lung parenchymal damage, incomplete resolution of pulmonary embolism, and endothelial dysfunction might cause persistent pulmonary hypertension [2], and as recommended by international guidelines, screening for chronic thromboembolic pulmonary hypertension will be performed at month 3 .

This case highlights the prothrombotic state of patients with SARS-CoV-2 infection and 


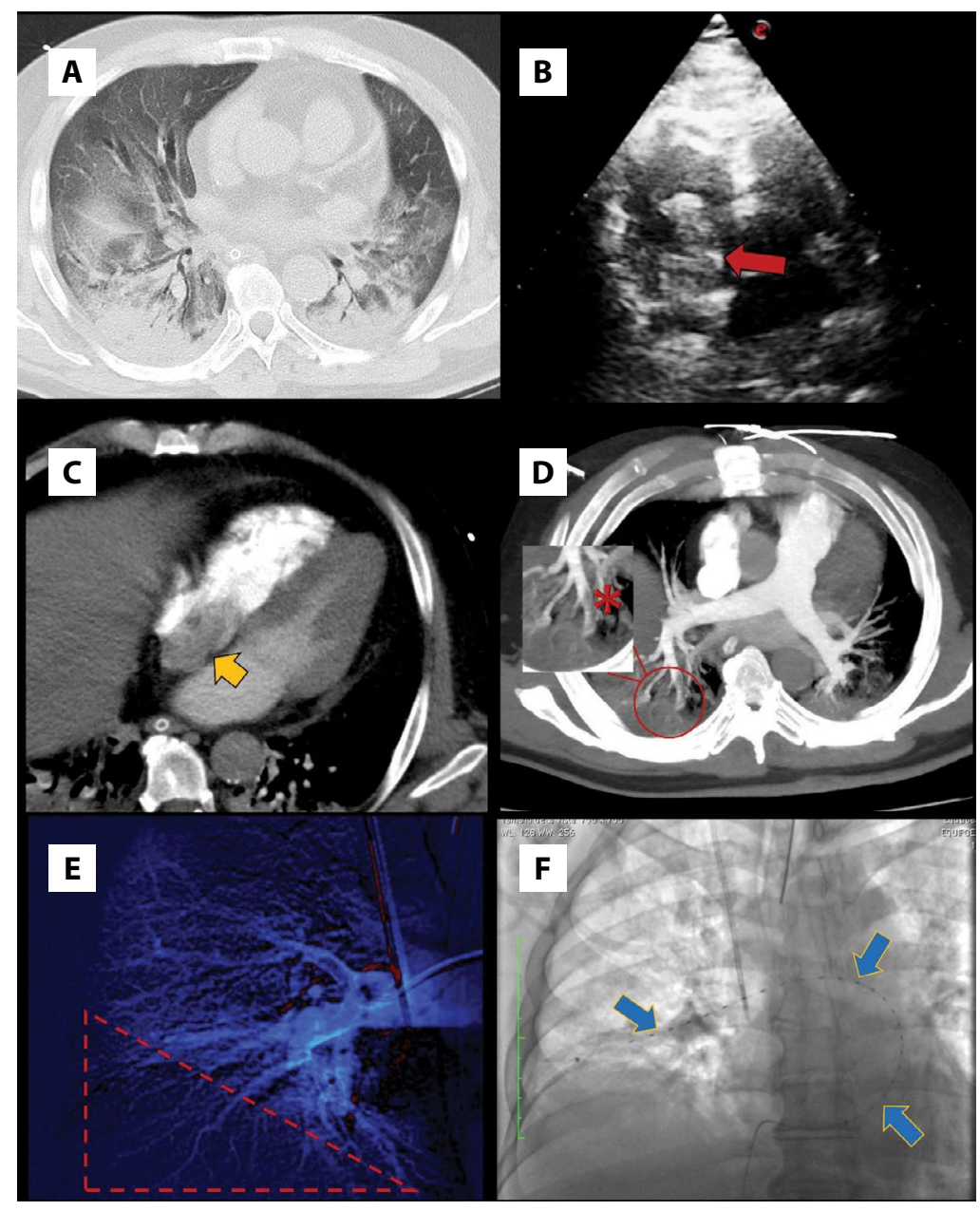

Figure 1. A. Chest computed tomography scan showing ground-glass opacities and crazy paving pattern in lower lobes associated with posterior basal consolidations. B. Transthoracic echocardiogram signalling the thrombus (red arrow). C. Four-chamber computed tomography signalling the thrombus (yellow arrow). D. Computed tomography pulmonary angiography demonstrating the pulmonary embolus (red asterisk). E. Pulmonary angiography demonstrating a hypoperfused area (dotted triangle). F. EKOS catheter inside the right atrium and right pulmonary artery (blue arrows)

demonstrates that ultrasound-assisted thrombolysis is an effective therapy for the treatment of concomitant right atrial thrombus and pulmonary embolism.

Advantages of ultrasound-assisted thrombolysis include the use of lower thrombolytic dose and lower bleeding risk compared with systemic thrombolysis [3]. Additionally, it is less invasive and more rapidly available compared with surgery [4], likely reducing the risk of exposure to a greater number of health personal in the operating and post-surgical care rooms. Potential benefits of a multidisciplinary pulmonary embolism response team strategy are improved outcomes, offering the most optimal strategy across a full range of advanced therapeutic options [5].

\section{Supplementary material}

Supplementary material is available at https://journals. viamedica.pl/kardiologia_polska.

\section{Article information}

Conflict of interest: None declared.

Open access: This article is available in open access under Creative Common Attribution-Non-Commercial-No Derivatives 4.0 International (CC BY-NC-ND 4.0) license, allowing to download articles and share them with others as long as they credit the authors and the publisher, but without permission to change them in any way or use them commercially. For commercial use, please contact the journal office at kardiologiapolska@ptkardio.pl.

How to cite: Victoria-Nandayapa JR, Arroyo-Rodríguez C, Franco-Rodríguez SL, et al. Ultrasound-assisted thrombolysis for a giant right atrial thrombus and pulmonary embolism in a COVID-19 patient. Kardiol Pol. 2021; 79(6): 710-711, doi: 10.33963/KP.15961.

\section{REFERENCES}

1. Francone M, lafrate F, Masci GM, et al. Chest CT score in COVID-19 patients: correlation with disease severity and short-term prognosis. Eur Radiol. 2020; 30(12): 6808-6817, doi: 10.1007/s00330-020-07033-y, indexed in Pubmed: 32623505.

2. Cruz-Utrilla A, Calderón-Flores M, Escribano-Subias MP. Pulmonary embolism and coronavirus disease 2019: persistent pulmonary hypertension? Kardiol Pol. 2020; 78(9): 937-938, doi: 10.33963/KP.15436, indexed in Pubmed: 32550733

3. Zarghouni M, Charles HW, Maldonado TS, et al. Catheter-directed interventions for pulmonary embolism. Cardiovasc Diagn Ther. 2016; 6(6): 651-661, doi: 10.21037/cdt.2016.11.15, indexed in Pubmed: 28123985.

4. Narang A, Mediratta A, Estrada JR, et al. Transcatheter therapy for a large mobile right atrial thrombus and massive pulmonary embolism. J Invasive Cardiol. 2016; 28(5): E49-E51, indexed in Pubmed: 27145056.

5. Sławek-Szmyt S, Jankiewicz S, Smukowska-Gorynia A, et al. Implementation of a regional multidisciplinary pulmonary embolism response team: PERT-POZ initial 1-year experience. Kardiol Pol. 2020; 78(4):300-310, doi: 10.33963/KP.15230, indexed in Pubmed: 32165606. 